PFC/JA-93-7

\title{
SPACE-TIME EVOLUTION OF \\ BEAM-PLASMA INSTABILITY IN STRONGLY CORRELATED PLASMAS
}

\author{
Z. C. Tao, A. K. Ram, A. Bers, and G. Kalman
}

May 1993

\begin{abstract}
Plasma Fusion Center
Massachusetts Institute of Technology

Cambridge, Massachusetts 02139 USA
\end{abstract}

This work was supported by NSF Grant Numbers: ECS-88-22475, ECS-87-13337, and PHY-91-15714 and DOE Grant No. DE-FG0291ER-54109. Reproduction, translation, publication, use and disposal, in whole or part, by or for the United States Government is permitted.

Submitted to: Physical Review E, Rapid Communication 


\section{SPACE-TIME EVOLUTION OF BEAM-PLASMA INSTABILITY IN STRONGLY CORRELATED PLASMAS}

Z. C. Tao, A. K. Ram, A. Bers, and G. Kalman

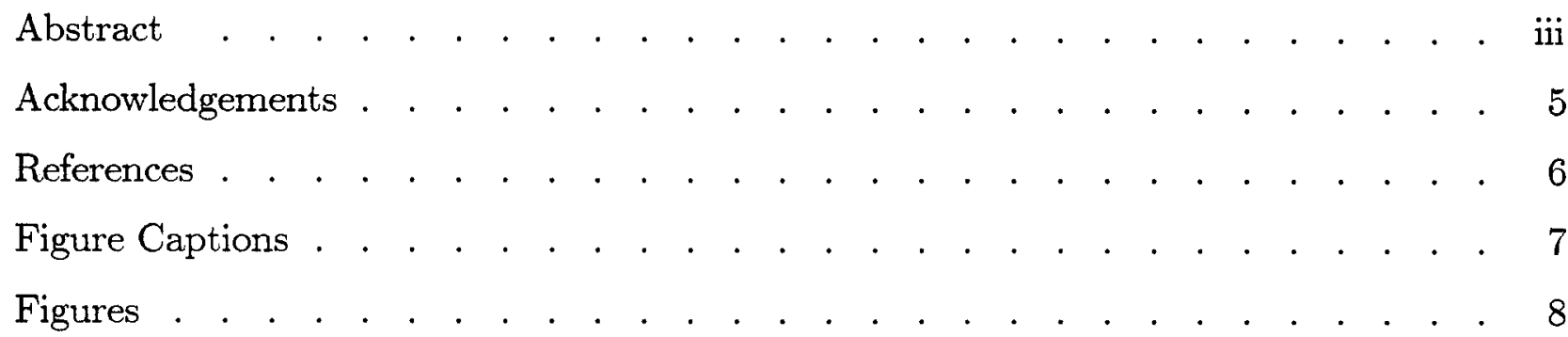




\title{
SPACE-TIME EVOLUTION OF BEAM-PLASMA INSTABILITY IN STRONGLY CORRELATED PLASMAS

\author{
Z.C. TAO ${ }^{1,2}$, A.K. RAM ${ }^{3}$, A. BERS 3 and G. KALMAN 1 \\ ${ }^{1}$ Department of Physics, Boston College \\ Chestnut Hill, MA 02167, USA \\ 2 Department of Physics, University of Western Ontario \\ London, Ontario N6A $3 K 7$, CANADA* \\ 3 Plasma Fusion Center, MIT \\ Cambridge, MA 02139, USA
}

\begin{abstract}
We examine the interaction of an electron beam penetrating a strongly correlated plasma. Conditions are established for this unstable interaction to lead to an absolute instability. We propose the absolute instability as a means for probing the strongly correlated plasmas.
\end{abstract}

April, 1993

PACS numbers: 52.35.Qz, 52.40.Mj, 52.25.Mq 
The collective mode structure of a strongly correlated (strongly coupled) plasma has characteristic features [1], distinguishing it from the mode structure of a weakly correlated system, which is well described by the Vlasov or random phase approximation. Most importantly the dispersion $(\partial \omega / \partial \mathrm{k})$ of the plasmon mode is diminished and becomes negative at a critical value of the coupling parameter. This latter represents the ratio of the potential to thermal energy and is conveniently given either as $\Gamma=e^{2} / \mathrm{k}_{\mathrm{B}} \mathrm{Ta}$ (a being the Wigner-Seitz radius) or as the plasma parameter $\gamma=1 / 4 \pi N_{D}$ defined through the number of electrons in the Debye cube $N_{D}=n / \kappa_{D}^{3}$, where $\kappa_{D}=\sqrt{4 \pi \beta n e^{2}}$ is the Debye wave number. The critical value of the plasma parameter $\gamma$ has been determined by computer simulations [2], by theoretical calculations $[3,4]$ and by experiments on alkali metals [5], converging to the value $\gamma=30 \sim 50$. When an electron beam penetrates a plasma, a beamplasma instability develops. In this Rapid Communication we point out that due to the change of the dispersion the space-time evolution of the beam-plasma instability changes character as the correlations become sufficiently strong.

The space-time evolution of the instability in an observer frame moving with velocity $\mathrm{V}$, in the non-relativistic limit, is described [6] by the Green function

$$
\begin{aligned}
G\left(x^{\prime}, t\right) & =\int_{L} \frac{d \omega^{\prime}}{2 \pi} \int_{F} \frac{d k}{2 \pi} e^{i\left(k x^{\prime}-\omega^{\prime} t\right)} \frac{1}{\varepsilon_{v}\left(k, \omega^{\prime}\right)} \\
& =\int_{L} \frac{d \omega}{2 \pi} \int_{F} \frac{d k}{2 \pi} e^{i(k x-\omega t)} \frac{1}{\varepsilon(k, \omega+k V)} .
\end{aligned}
$$

Here $\mathrm{x}^{\prime}=\mathrm{x}+\mathrm{Vt} . ; \varepsilon(\mathrm{k}, \omega)$ is the dielectric function for the system under consideration. $\mathrm{L}$ and $\mathrm{F}$ are the Laplace and Fourier contours in the $\omega$ and $\mathrm{k}$ planes, respectively. The behavior of the Green function $G\left(x^{\prime}, t\right)$ is totally determined by the analytic nature of the 
dielectric function $\varepsilon(k \omega)$. The time asymptotic form of the Green function gives the pulse shape in the laboratory frame. It is known [6] that the pulse shape in the laboratory frame can be deduced from the analysis of the pinch point $\left(\mathrm{k}_{0}, \omega_{0}\right)$ condition:

$$
\varepsilon\left(\mathrm{k}_{0}, \omega_{0}+\mathrm{k}_{0} \mathrm{~V}\right)=0 ; \frac{\partial}{\partial \mathrm{k}} \varepsilon\left(\mathrm{k}_{0}, \omega_{0}+\mathrm{k}_{0} \mathrm{~V}\right)=0
$$

We consider the interaction of an electron beam penetrating a strongly correlated plasma. We model the present beam-plasma system by two plasmas in relative motion $\mathrm{v}_{0}$ with different temperatures $1 / \beta_{b}$ and $1 / \beta$, and different densities $n_{b}$ and $n$. Hence, these two plasmas have two different plasma parameters $\gamma_{b}$ and $\gamma$. The dielectric function for the present beam-plasma system is given by

$$
\begin{aligned}
\varepsilon(\mathrm{k}, \omega)= & 1-\frac{\varphi(\mathrm{k}) \chi_{0}(\mathrm{k}, \omega)}{1+\mathfrak{G}(\mathrm{k}, \omega) \varphi(\mathrm{k}) \chi_{0}(\mathrm{k}, \omega)} \\
& -\frac{\varphi(\mathrm{k}) \chi_{0 \mathrm{~b}}\left(\mathrm{k}, \omega-\mathrm{kv}_{0}\right)}{1+\mathfrak{G}_{\mathrm{b}}\left(\mathrm{k}, \omega-\mathrm{kv}_{0}\right) \varphi(\mathrm{k}) \chi_{0 \mathrm{~b}}\left(\mathrm{k}, \omega-\mathrm{kv}_{0}\right)}
\end{aligned}
$$

with $\chi_{0}(\mathrm{k}, \omega)$ and $\mathcal{G}(\mathrm{k}, \omega)$ denoting the non-interacting Vlasov density response function and the dynamical local field, respectively; $\varphi(\mathrm{k})=4 \pi \mathrm{e}^{2} / \mathrm{k}^{2}$ is the Coulomb potential. The dynamical local field describes the correlational contribution to the dielectric function, as formulated in Refs. [1,4,7]. In the present paper, we will only consider a special case where the electron beam is weakly correlated $\gamma_{\mathrm{b}} \ll 1$ and its temperature is much less than that of the strongly correlated plasma $\beta / \beta_{b}<<1$. Then the dielectric function reduces to

$$
\varepsilon(\mathrm{k}, \omega)=1-\frac{\mathrm{b}}{\left(\omega-\mathrm{kv}_{0}\right)^{2}}-\frac{\varphi(\mathrm{k}) \chi_{0}(\mathrm{k}, \omega)}{1+\mathfrak{G}(\mathrm{k}, \omega) \varphi(\mathrm{k}) \chi_{0}(\mathrm{k}, \omega)}
$$


Here $b=n_{b} / n$, and $k, v$, and $\omega$ are normalized to $\kappa_{D}$, the electron thermal velocity $v_{\mathrm{T}}=\sqrt{1 / \mathrm{m} \beta}$, and the plasma frequency $\omega_{\mathrm{p}}=\sqrt{4 \pi \mathrm{ne}^{2} / \mathrm{m}}$, respectively. The zeros of this dielectric function are given by

$$
\left(\omega-\mathrm{kv}_{0}\right)^{2}\left[\mathrm{k}^{2}-\mathrm{Z}(\omega / \mathrm{k})+\mathfrak{G}(\mathrm{k}, \omega) Z(\omega / \mathrm{k})\right]=\mathrm{b}\left[\mathrm{k}^{2}+\mathfrak{G}(\mathrm{k}, \omega) Z(\omega / \mathrm{k})\right]
$$

with $\mathrm{Z}$ denoting the plasma dispersion function [9]. In the long wavelength limit, i.e., $\mathrm{k} / \mathrm{K}_{\mathrm{D}} \ll 1$, the above equation becomes

$$
\left(\omega-\mathrm{kv}_{0}\right)^{2}\left[\omega^{2}-1-2 \alpha \mathrm{k}^{2}+2 i \eta \omega \mathrm{k}^{2}\right]=\mathrm{b}\left[\omega^{2}-(2 \alpha-3) \mathrm{k}^{2}+2 \mathrm{i} \eta \omega \mathrm{k}^{2}\right]
$$

where $\alpha$ and $\eta$ essentially determine the plasmon group velocity and damping. $\alpha$ and $\eta$ as a function of the plasma parameter $\gamma$ have been calculated $[3,4]$; the results presented here (Fig. 1) are based on the calculation of Ref. [4]. The warm Vlasov plasma is obtained in the limit $\gamma \rightarrow 0$ where $\alpha=1.5$ and $\eta=0$. As $\gamma$ increases, the plasmon group velocity decreases. Above the critical value $\gamma \geq 50$, the plasmon group velocity becomes negative. The plasmon damping increases as $\gamma$ increases.

The interaction between the beam waves and the plasma waves in the strongly coupled plasma, as shown in Fig. 2, is described by Eq. (6). As can be seen, in the strongly correlated plasma where $\gamma>\gamma_{\text {crit }}$, the plasmon group velocity can become opposite to that of the electron beam waves, thus giving rise to the possibility of an absolute instability. The absolute instability results from the phase resonant interaction between the forward beam waves and backward plasma waves. We now establish the conditions for the beam-plasma instability to become absolute.

We begin with an analysis of the solutions to Eq. (6) for the real $\underline{k}$. The results are presented in the complex $\omega$ plane in Fig. 3. The branch with $\omega_{i}>0$ indicates the beamplasma system exhibits an instability. The maximum growth of this instability occurs when 
condition $\left|\mathrm{kv}_{0}\right| \sim 1$ is met. Note that Eq. (6) is an adequate representation to Eq. (5) only in the long wavelength limit. Hence, in order for our above analysis and the following to be valid, the velocity of the electron beam should be much greater than the thermal velocity of the strongly correlated plasma.

We employ the pinch point analysis [6] to determine the nature of the space-time evolution for the present beam-plasma system. The pinch point analysis consists of mapping the complex $\omega$ plane into the complex $\underline{\mathrm{k}}$ plane. Through this analysis, the solutions to Eq. (2) can be easily determined. It is obvious from Eqs. (2), (5) and (6) that the asymptotic form of the Green function is influenced by the plasmon group velocity $\alpha$ and damping $\eta$. The damping in the strongly correlated situation is almost exclusively collisional, moderated by the increased tendency of the particles to localize and to avoid their neighbors; Landau damping is negligible. We have performed the pinch point analysis for the present beam-plasma system: it is found that lowering $\alpha$ moves the trailing edge of the pulse shape to the negative direction, while increasing $\eta$ moves the trailing edge toward the opposite direction. Hence, under favorable conditions, the trailing edge of the pulse shape lies in the negative side of the origin, and the beam-plasma instability becomes absolute [6]. In Fig. 4, we present the condition for $v_{0}=10$ and $b=0.001$. In the $\alpha-\eta$ plane, these conditions form a boundary line separating convective instability from absolute instability for the present beam-plasma system.

In the strongly correlated plasma, $\alpha$ and $\eta$ are determined by the plasma parameter $\gamma$. Plotting $\alpha$ and $\eta$ as a function of $\gamma$ yields another curve in Fig. 4. We find that the present curve intersects with the boundary curve which separates convective from absolute instabilities at the point corresponding to $\gamma \sim 60$. Calculations based on other analyses of the plasmon dispersion $[1,3]$ would give somewhat lower, but not substantially different results. Therefore, for $\gamma>60$, the beam-plasma instability in strongly correlated plasmas is absolute for the present choice of the parameters. 
As mentioned earlier, the pinch point analysis not only distinguishes convective from absolute space-time evolution, but also yields the asymptotic form of the Green function $G\left(x^{\prime}, t\right)$ or the pulse shape. We present the pulse shape for the beam-plasma system in the weakly and strongly correlated plasmas in Fig. 5. As can be seen, the trailing edges of the pulse shape in the weakly correlated plasma such as $\gamma=0$ and in the strongly correlated plasma such as $\gamma=87$ lie in the opposite sides of the origin, revealing the nature of the space-time evolution for the corresponding beam-plasma system. The leading edges, however, are in the same place despite the differences between these two different plasmas. As demonstrated in a previous study [10], the leading edges always reside in the same place as long as the beam plasma is cold. It has been shown that the thermal motion in the electron beam would slow down the leading edge. Hence, this would not interfere with the nature of the space-time evolution of the present beam-plasma system.

To summarize, we find that electron correlations profoundly affect the manner the beam-plasma system evolves with time. As the plasma parameter increases, the nature of the beam-plasma instability is changed from convective to absolute, at a critical value of the plasma parameter. This phenomenon may provide a useful experimental approach to probe and identify strongly correlated plasmas.

\section{ACKNOWLEDGMENTS}

This work was partially supported by the National Science Foundation Grants No. ECS-87-13337, and PHY-91-15714. ZCT acknowledges the support of his colleagues at UWO during the final stage of the work. This work was also partially supported by the National Science Foundation Grant No. ECS-88-22475 and the Department of Energy Grant No. DE-FG02-91ER-54109. 


\section{REFERENCES}

* present address. E-mail address: tao@uwovax.uwo.ca.

[1] G. Kalman, in "Condensed Matter Theories 7 ", edited by A.N. Proto and J.L. Aliaga, (Plenum Press, N.Y., 1991).

[2] J.P. Hansen, E.L. Pollock and I.R. McDonald, Phys. Rev. 11A, 1025 (1975).

[3] P. Carini, G. Kalman and K.I. Golden, Phys. Lett. 78A, 450 (1980).

P. Carini and G. Kalman, Phys. Lett. 105A, 229 (1984).

M. Minella, G. Kalman, K.I. Golden and P. Carini, submitted for publication.

[4] Z.C. Tao, Ph.D. Thesis, Boston College, 1990 (unpublished).

[5] A. VonFelde, J. Sprosser-Prou and J. Fink, Phys. Rev . B40, 10181 (1989).

[6] A. Bers, in Handbook of Plasma Physics, edited by M.W. Rosenbluth and

R.Z. Sagdeev, Basic Plasma Physics, vol. 1, edited by A.A. Galeev and

R.N. Sudan (North-Holland, Amsterdam, 1983), Chap. 3.2.

[7] S. Ichimaru, Rev. Mod. Phys. 54, 1017 (1982).

[8] Z.C. Tao and G. Kalman, Phys. Rev. 43A, 973 (1990).

[9] B.D. Fried and S. Conte, The Plasma Dispersion Function (Academic Press, New York, 1961).

[10] G. Francis, A.K. Ram, and A. Bers, Phys. Fluids 29, 255 (1986). 


\section{FIGURES}

FIG. 1. The plasmon group velocity $\alpha$ and damping $\eta$ as a function of the plasma parameter $\gamma$.

FIG. 2. Rew vs. Rek, as given by Eq.(6). A and B denote Rew for the weakly $(\gamma=0)$ and strongly $(\gamma=87)$ correlated plasmas; $\mathrm{C}$ gives the beam wave.

FIG. 3. Numerical solutions to Eq.(6) in the $\omega$ plane for $0.0<k<0.2$ for $\gamma=87$.

FIG. 4. Convective-absolute boundary in the $\alpha-\eta$ plane, together with the results for $\alpha$ and $\eta$ as a function of $\gamma$ (dashed curve).

FIG. 5. The pulse shape for the beam-plasma system. Solid and dashed curves give the pulse shape in the strongly $(\gamma=87)$ and weakly $(\gamma=0)$ correlated plasmas. 


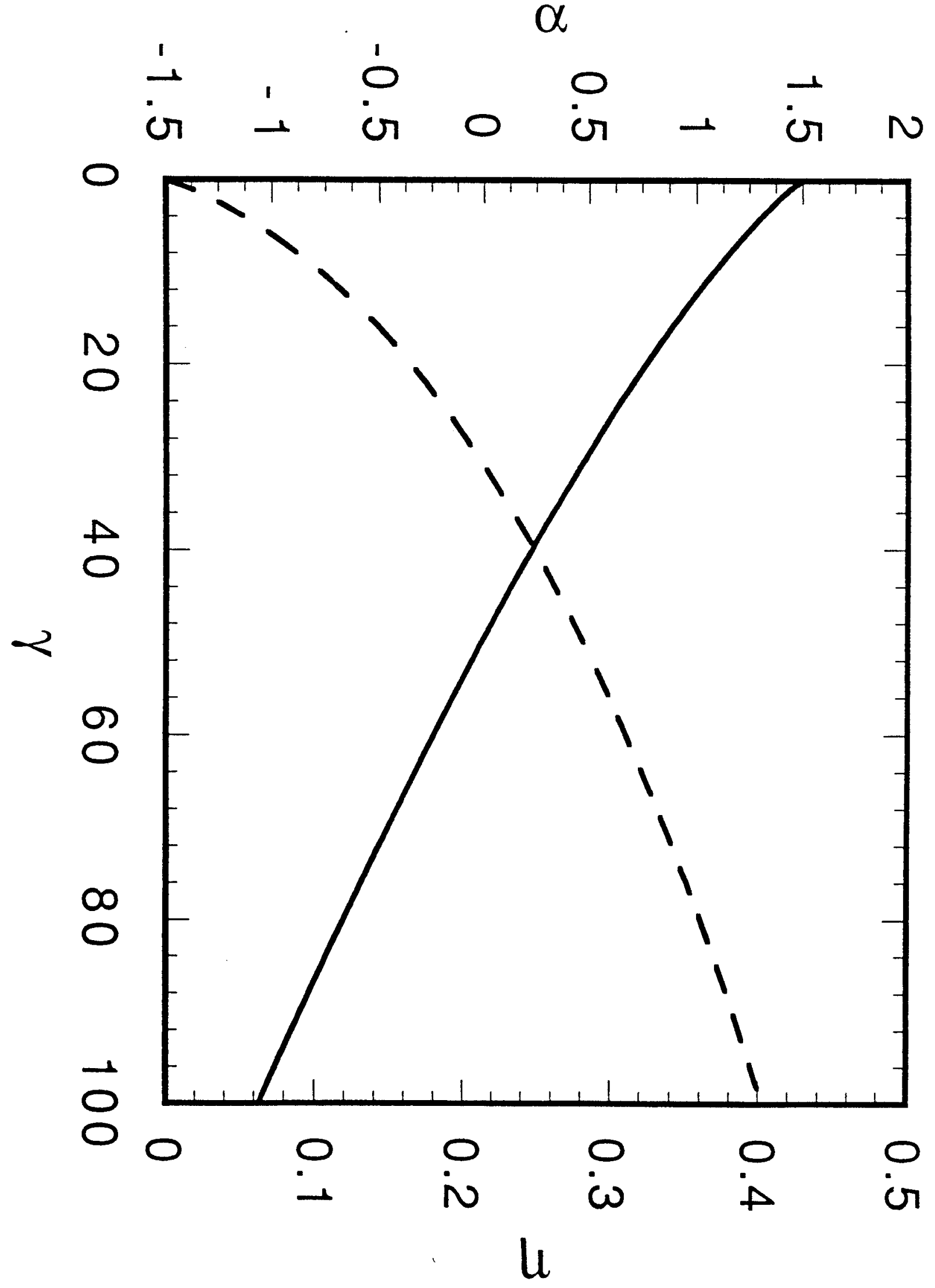




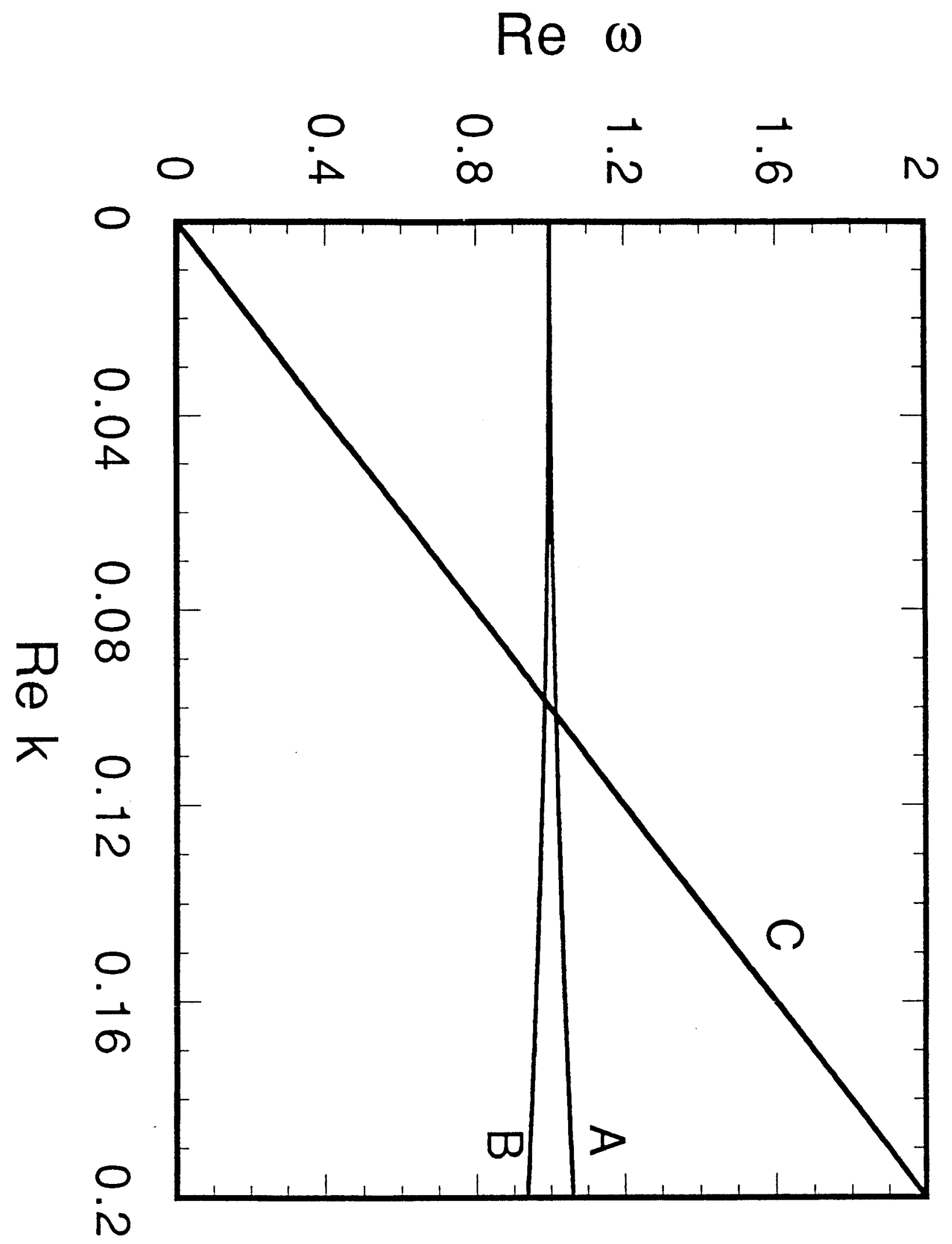




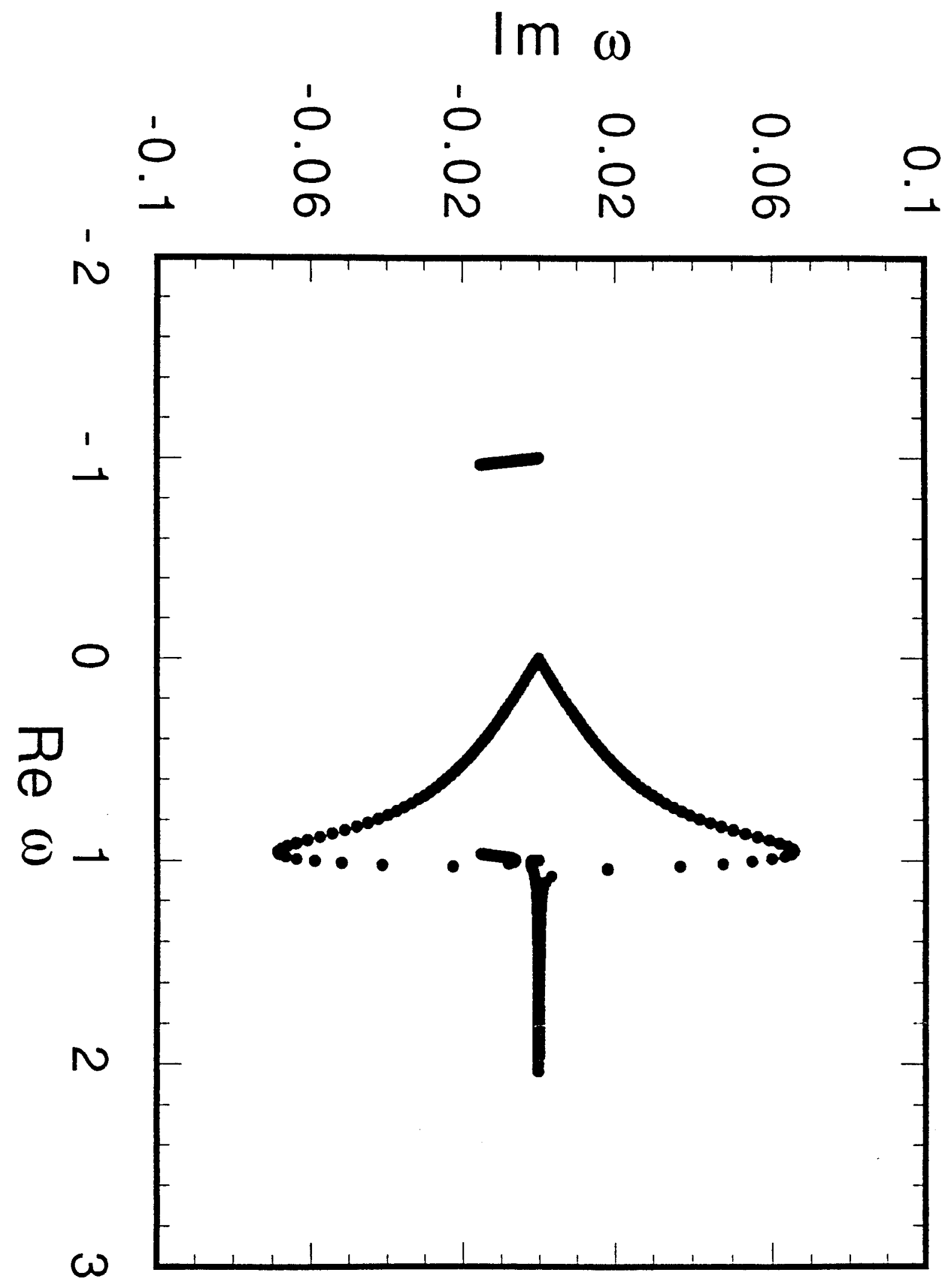


$\eta$

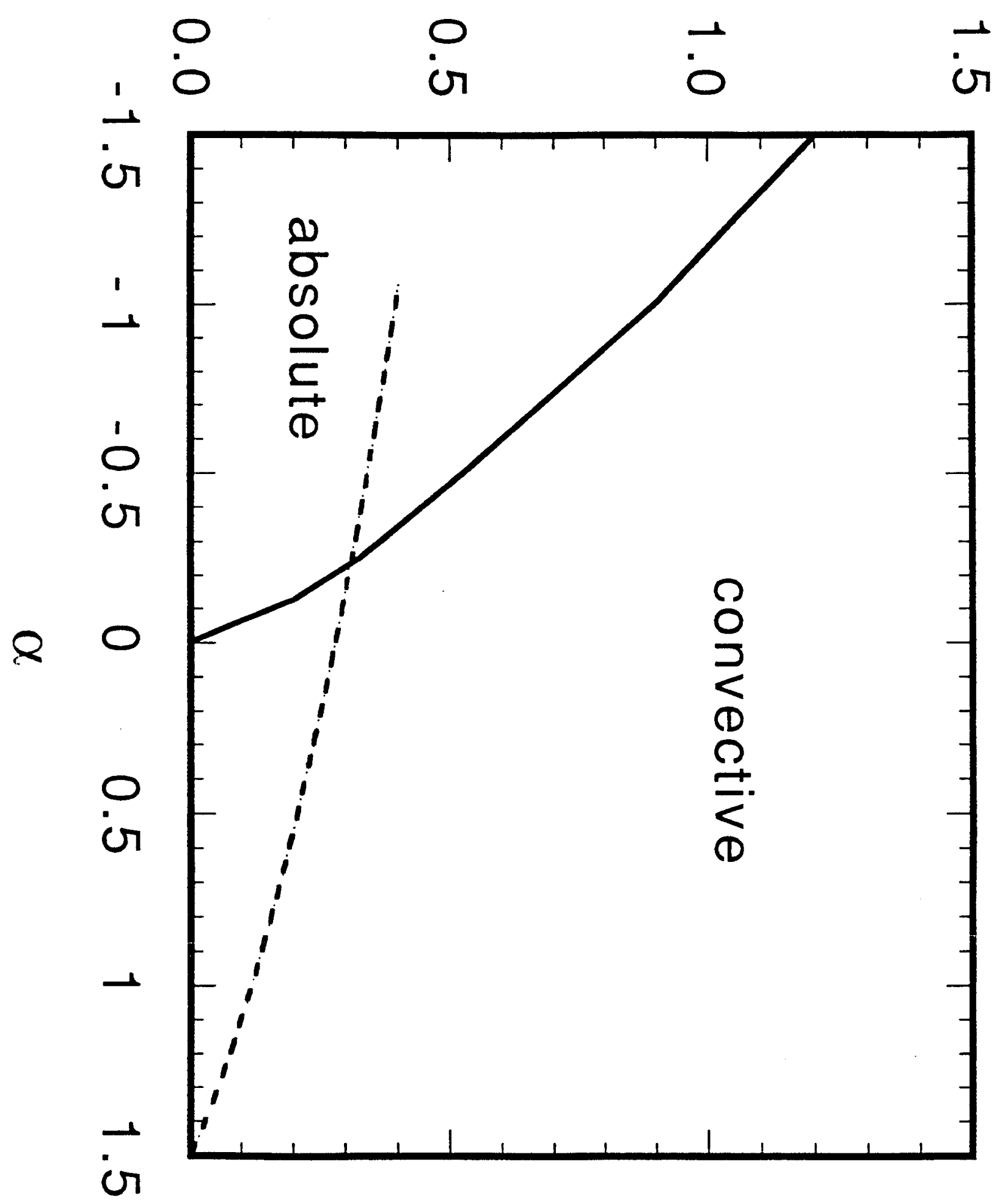




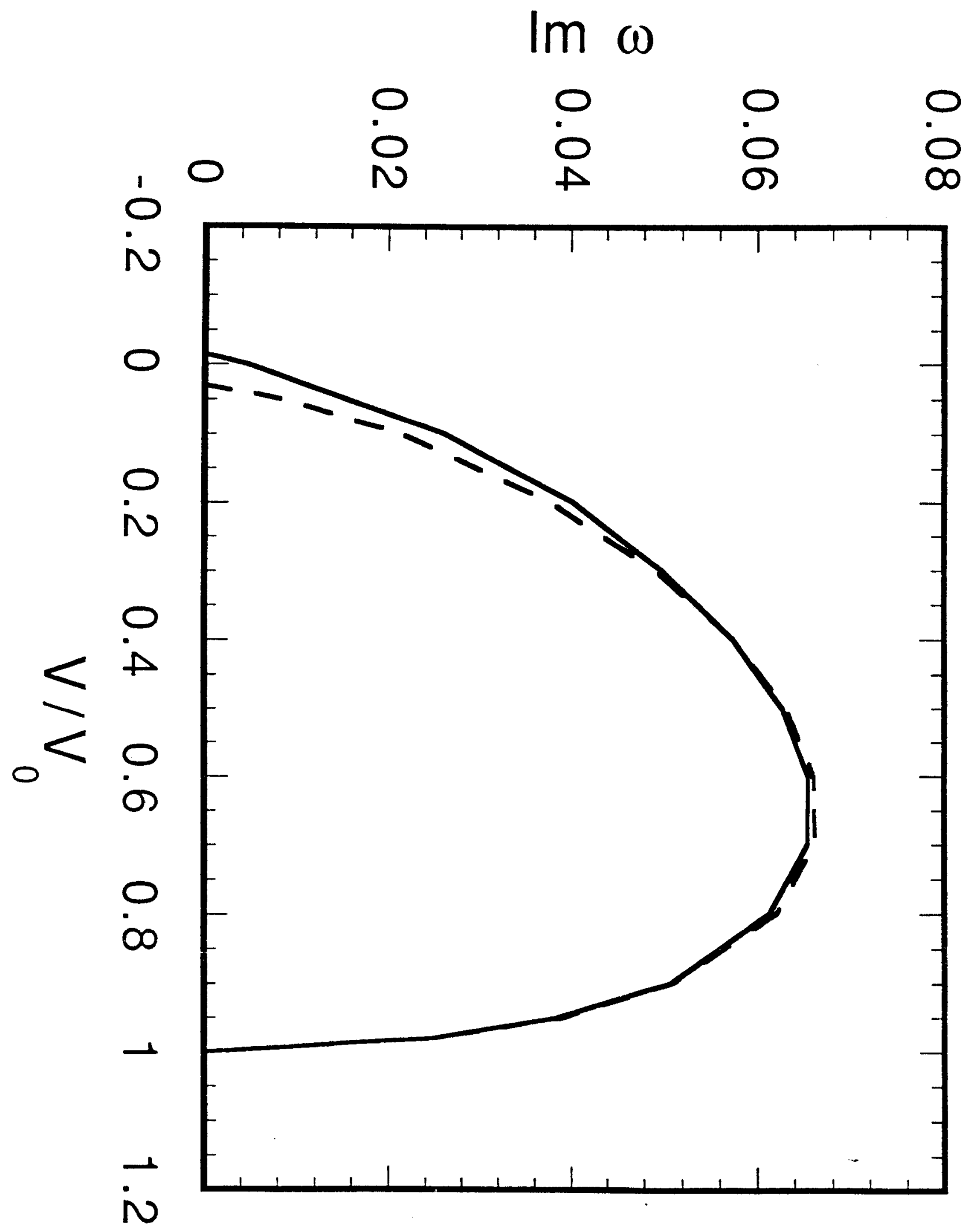

